\title{
Paradoxical low-flow aortic stenosis - baseline characteristics, impact on mortality
}

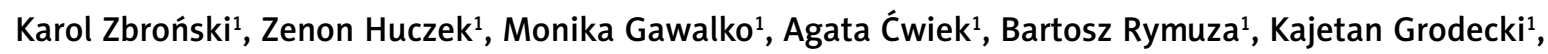 \\ Piotr Scisło ${ }^{1}$, Radosław Wilimski ${ }^{2}$, Janusz Kochman ${ }^{1}$, Krzysztof J. Filipiak ${ }^{1}$, Grzegorz Opolski ${ }^{1}$ \\ ${ }^{1} 1^{\text {st }}$ Chair and Department of Cardiology, Medical University of Warsaw, Warsaw, Poland \\ 2Department of Cardiac Surgery, Medical University of Warsaw, Warsaw, Poland
}

Adv Interv Cardiol 2019; 15, 1 (55): 13-19

DOI: https://doi.org/10.5114/aic.2019.83770

\begin{abstract}
Introduction: Paradoxical low-flow, low-gradient aortic stenosis (pLF-LGAS) constitutes an important part of the population undergoing transcatheter aortic valve implantation (TAVI). However, it remains the least defined subtype of aortic stenosis (AS).

Aim: To investigate baseline characteristics and impact on mortality of pLF-LGAS in patients treated with TAVI.

Material and methods: Two-hundred and thirty-one consecutive patients (mean aortic valve area: $0.76 \pm 0.41 \mathrm{~cm}^{2}$ ) who underwent TAVI in our centre during the period of 6 years were included in the study. Based on echocardiographic examination, patients with pLF-LGAS were identified, analysed and compared to a population with high-gradient AS (HGAS) and low-flow, low-gradient AS with reduced ejection fraction (classical, CLF-LGAS). The primary endpoints of the study were all-cause mortality after 30 days and 1 year.

Results: pLF-LGAS was diagnosed in 42 (18.2\%) patients, whereas 40 (17.3\%) had CLF-LGAS and 149 (64.5\%) had HGAS. The pLF-LGAS population was younger, had higher prevalence of hypertension, and had higher ejection fraction (EF) than the HGAS population, and had a smaller proportion of heavily symptomatic patients than the cLF-LGAS population. Overall, 46 (19.9\%) patients died within 12 months after TAVI. The 30-day and 1-year survival was comparable between AS subtypes. Multivariate analysis identified severe renal failure as an independent predictor of mortality among all patients.

Conclusions: pLF-LGAS is common among subjects undergoing TAVI. Patients with paradoxical AS are younger, more often burdened with hypertension and have higher EF than the HGAS population, while being less symptomatic than the CLF-LGAS group. Presence of pLF-LGAS does not seem to affect short- and mid-term survival. Severe renal failure is an independent predictor of mortality after TAVI.
\end{abstract}

Key words: aortic stenosis, long-term mortality, transcatheter aortic valve implantation.

S u m m a ry

Paradoxical low-flow, low-gradient aortic stenosis (pLF-LGAS) is common among subjects undergoing transcatheter aortic valve implantation (TAVI). Patients with paradoxical AS are younger, more often burdened with hypertension and have higher ejection fraction than the high-gradient AS (HGAS) population, while being less symptomatic than the low-flow, low-gradient AS with reduced ejection fraction (LF-LGAS) group. Presence of pLF-LGAS does not seem to affect short- and mid-term survival. Severe renal failure is an independent predictor of mortality after TAVI.

\section{Introduction}

Aortic stenosis (AS) is the most common acquired valvular heart disease in Europe and North America, with about $5 \%$ of people aged above 65 suffering from this disease and the mean survival estimated at two to 5 years depending on the severity of symptoms [1, 2].
Transcatheter aortic valve replacement (TAVI) is the treatment of choice in patients who are not suitable for surgical aortic valve replacement (AVR) or are at increased surgical risk [3-5]. A special group of patients undergoing TAVI is subjects with low-flow, low-gradient AS (LF-LGAS). They pose a diagnostic and therapeutic challenge, being distinctly different from the majority of patients with

Corresponding author:

Monika Gawalko, $1^{\text {st }}$ Chair and Department of Cardiology, Medical University of Warsaw, 1 a Banacha St, 02-097 Warsaw, Poland, phone: +48 664909 939, e-mail: mongawalko@gmail.com

Received: 21.10.2018, accepted: 27.12.2018. 
high gradient AS (HGAS) [3-5]. Current American and European guidelines distinguish 2 subtypes of LF-LGAS [3-5] - with reduced left ventricle ejection fraction (LVEF; classical, CLF-LGAS), most often due to ischaemic heart disease, and with normal LVEF (paradoxical, pLF-LGAS), in large part associated with restrictive physiology and a history of hypertension. The latter in particular remains a diagnostic conundrum, with various investigators reporting different baseline characteristics and a varied impact on mortality of this phenomenon.

\section{Aim}

Therefore, the aim of this study was to further investigate the nature of paradoxical LF-LGAS in patients treated with TAVI.

\section{Material and methods}

\section{Study population}

The current study represents a retrospective analysis of 231 consecutive patients who underwent TAVI at our institution from March 2010 to March 2016. According to the 2012 and 2017 European and 2014 American guidelines for the management of valvular heart disease, only patients with symptomatic severe AS were enrolled for a TAVI procedure [3-5]. Patients were followed up for 12 months. Written informed consent for all studies and TAVI was obtained from all patients. All demographic, clinical, laboratory, and echocardiographic data were retrieved retrospectively from medical records. The Institutional Review Committee on Human Research agreement was not required due to the retrospective character of the study.

\section{Echocardiographic measurement}

All patients underwent standard 2-dimensional B mode and Doppler transthoracic echocardiography before the procedure in our department's Echocardiography Laboratory (certified with grade $C$ accreditation of the Section of Echocardiography of the Polish Cardiac Society) on an EPIQ 7 Ultrasound Machine (Philips Medical Systems, Andover, MA, USA) or on an iE33 Ultrasound Machine (Philips Medical Systems, Andover, MA, USA), using a Philips X7-2t TOE ultrasound transducer (Philips Medical Systems, Andover, MA, USA). The conventional parameters were measured according to the available guidelines [3-5]. Left ventricular ejection fraction assessment was performed using modified biplane Simpson's method from the apical two- and four-chamber view. Aortic valve area (AVA) was measured using the continuity equation according to current guidelines and indexed to body surface area (AVAi). Transaortic mean pressure gradient (MPG) was obtained according to the Bernoulli equation. Stroke volume was measured by pulsed wave Doppler in the left ventricle (LV) outflow tract and was indexed for body surface area (SVI). Following guidelines [3], patients with symptomatic severe AS (AVA $<1 \mathrm{~cm}^{2}$, $A V A i<0.6 \mathrm{~cm}^{2}$ ) were retrospectively grouped according to mean transvalvular pressure gradient $(40 \mathrm{~mm} \mathrm{Hg}$ as cutoff) into LGAS and HGAS groups. Among the LGAS group, only patients with low-flow stenosis (i.e. SVI $<35 \mathrm{ml} / \mathrm{m}^{2}$ ) underwent TAVI and were included in the analysis, while in patients presenting normal flow, AS was deemed unlikely as the cause of symptoms. The process of identifying patients with true severe AS was performed case-by-case by a team encompassing an experienced echocardiographer, two cardiac interventionalists and a radiologist and based on clinical data as well as additional factors such as morphology and calcifications of the aortic valve in the transthoracic echocardiography, transesophageal echocardiography, calcium score by multi-slice computed tomography as well as the low-dose dobutamine test. All patients included in the analysis were deemed to have true severe AS. For subanalysis, the LF-LGAS group was split into two groups: LF-LGAS with reduced (LVEF < 50\%) and preserved ejection fraction (LVEF > 50\%). The study flow chart is presented in Figure 1.

\section{Complications}

According to Bleeding Academic Research Consortium (BARC), major bleeding was defined as overt bleeding either associated with a drop in the haemoglobin level of at least $3.0 \mathrm{~g} / \mathrm{dl}$ or requiring transfusion of two or three units of whole blood/red blood cells (RBC), or causing hospitalization or permanent injury, or requiring surgery (BARC type $3 \mathrm{a}$ ), and did not meet criteria of life-threatening bleeding. Life-threatening bleeding was defined as fatal bleeding (BARC type 5) or pericardial bleeding necessitating pericardiocentesis, or intramuscular with compartment syndrome (BARC type $3 b$ and $3 c$ ) or bleeding causing hypovolaemic shock or severe hypotension requiring vasopressors or surgery or bleeding with a drop in haemoglobin $\geq 5 \mathrm{~g} / \mathrm{dl}$ or whole blood or packed RBC transfusion $\geq 4$ units (BARC type $3 b$ ) [6].

\section{Statistical analysis}

Continuous variables are presented as mean \pm standard deviation and were compared using the Mann-Whitney $U$ test for two-group comparison and the ANOVA test for multi-group comparison with posthoc Bonferroni and Games-Howell tests depending on the equality of variances. Categorical variables are presented as absolute numbers (percentages) and were compared using the $\chi^{2}$ or Fisher's exact test as appropriate. A value of $p<0.05$ was considered statistically significant, except in cases where Bonferroni's correction for multigroup comparisons was applied. All tests were two-tailed. Statistical analyses were performed using SPSS software, version 22 (IBM SPSS Statistics 22, USA, New York). 


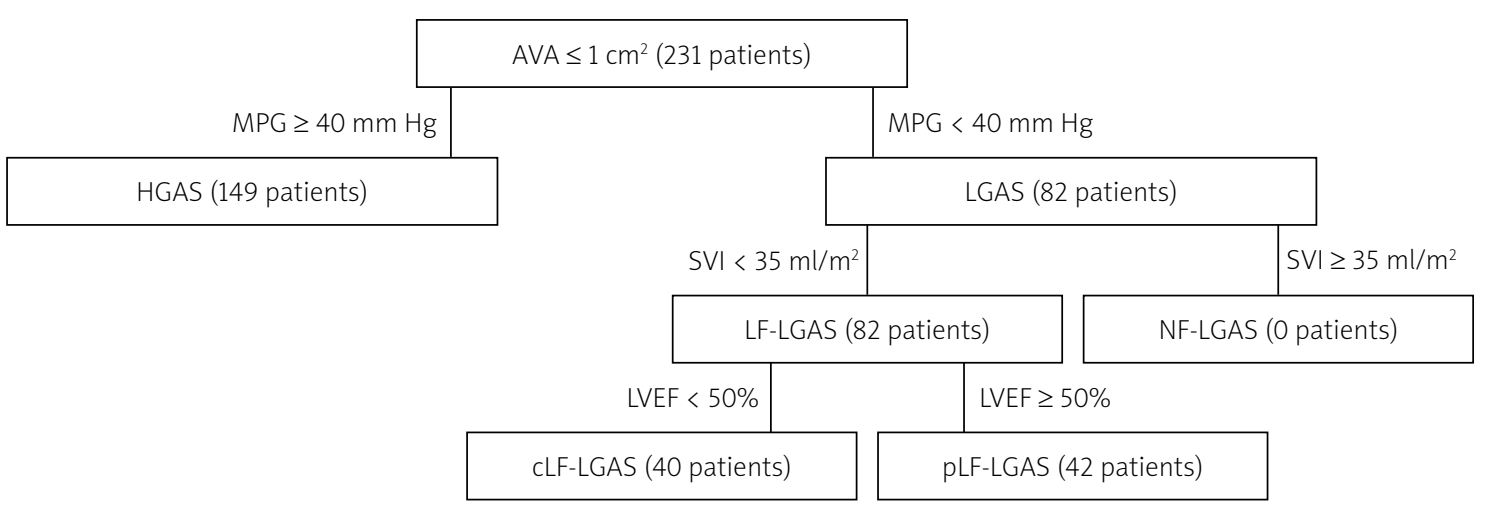

Figure 1. Study flow chart

AVA - aortic valve area, CLF-LGAS - classical low-flow, low-gradient aortic stenosis, HGAS - high-gradient aortic stenosis, LVEF - left ventricle ejection fraction, MPG - mean pressure gradient, pLF-LGAS - paradoxical low-flow, low-gradient aortic stenosis, SVI-stroke volume indexed for body surface area.

\section{Results}

\section{Baseline characteristics}

Baseline clinical and echocardiographic characteristics with subdivision of patients into the three defined types of AS are displayed in Table I. Among the 231 patients, 42 (18.2\%) were diagnosed with pLF-LGAS, 149 (64.5\%) with HGAS and 40 (17.3\%) with CLF-LGAS. The total cohort (49.8\% men, $50.2 \%$ women) was characterized by advanced age (79.5 \pm 7.2 years) and numerous comorbidities. The PLF-LGAS population was younger than the HGAS group (77.28 \pm 8.14 vs. $80.54 \pm 6.7, p=0.03$ ), had higher prevalence of hypertension than the CLF-LGAS group ( $90.5 \%$ vs. $62.5 \%, p=0.01)$, had higher $\mathrm{EF}$ than the HGAS group (59.71 \pm 6.5 vs. $54.48 \pm 12.8, p=0.03)$ and had a smaller proportion of heavily symptomatic patients than the CLF-LGAS group ( $50 \%$ vs. $82.5 \%$ of patients in NYHA class III or greater, $p=0.01$ ).

\section{Procedural characteristics}

The majority of the patients underwent TAVI using a trans-femoral approach (81.8\%) and self-expandable valves (67.1\%) including: Medtronic CoreValve/Evolut $\mathrm{R}$ (Medtronic, Minneapolis, MN), Lotus (Boston Scientific), JenaValve (JenaValve Technology GmbH, Munich, Germany) and Portico (St. Jude Medical, Minneapolis, MN, USA) prostheses. Total procedure and fluoroscopy times were 190 and $31 \mathrm{~min}$, respectively. The main periprocedural TAVI complications included major and life-threatening bleeding (10\%) and stroke/transient ischaemic attack (TIA) (4.8\%). There were no statistically significant procedural differences between subgroups. Detailed procedural characteristics of the study group are presented in Table I.

\section{Clinical outcomes}

Overall, 46 (19.9\%) patients died during the follow-up period of 12 months. Distilled, the mortality rate among patients with pLF-LGAS, HGAS and CLF-LGAS was 6 (14.3\%), 31 (20.8\%) and 9 (22.5\%), respectively. Kaplan-Meier sur- vival curve comparison did not demonstrate significant differences between the groups in the 30-day $(p=0.61)$ or one-year follow-up ( $p=0.62$ ) (Figure 2). Multivariate analysis identified severe renal failure (RF) as the only independent predictor of survival among AS patients (odds ratio $(\mathrm{OR})=2.1,95 \%$ confidence intervals $(\mathrm{Cl}): 1.01-4.37$, $p=0.046$, Table II).

\section{Discussion}

\section{Paradoxical low-flow, low-gradient stenosis \\ Baseline characteristics}

A considerable portion of papers investigating the matter of pLF-LGAS originate from the surgical world and suggest that pLF-LGAS corresponds to a more advanced stage of the disease as reflected by more severe intrinsic myocardial damage and the appearance of a restrictive physiology [7-9]. This state is postulated to share many pathophysiological and clinical similarities with preserved LVEF heart failure, with prevalence increasing with older age, female gender, and concomitant systemic arterial hypertension. In the study by Clavel et al., compared to cLF-LGAS, patients with pLF-LGAS undergoing AVR were more frequently female and had lower prevalence of comorbidities such as chronic obstructive pulmonary disease (COPD), coronary artery disease (CAD) and chronic kidney disease (CKD) [10]. Lopez-Marco et al. documented a higher proportion of females, greater incidence of hypertension and previous neurologic events as well as lower prevalence of diabetes and COPD in patients with pLF-LGAS undergoing AVR as compared to those with CLF-LGAS [11]. In other studies, the comorbidity profile of pLF-LGAS patients did not differ from HGAS patients with comparable age, occurrence of hypertension, diabetes, obesity, CAD and previous MI [9, 12].

In our study, a number of differentiating aspects were identified. Patients with pLF-LGAS were significantly younger than those with HGAS, had a noticeably higher prevalence of hypertension than the cLF-LGAS group, 
Table I. Characteristics of study population

\begin{tabular}{|c|c|c|c|c|c|}
\hline Parameter & Total $(n=231)$ & pLF-LGAS $(n=42)$ & HGAS $(n=149)$ & cLF-LGAS $(n=40)$ & $P$-value \\
\hline \multicolumn{6}{|l|}{ Baseline characteristics: } \\
\hline Age [years] & $79.5 \pm 7.2$ & $77.3 \pm 8.14^{a}$ & $80.5 \pm 6.7^{c}$ & $78.1 \pm 7.2$ & 0.012 \\
\hline Female sex & $116(50.2)$ & $23(54.8)$ & $81(54.4)$ & $12(30)$ & 0.019 \\
\hline $\mathrm{BMI}\left[\mathrm{kg} / \mathrm{m}^{2}\right]$ & $27.0 \pm 5.0$ & $26.8 \pm 5$ & $27.3 \pm 5.2$ & $25.9 \pm 4$ & 0.29 \\
\hline $\mathrm{BSA}$ & $1.80 \pm 0.2$ & $1.81 \pm 0.2$ & $1.79 \pm 0.2$ & $1.82 \pm 0.2$ & 0.67 \\
\hline EuroSCORE logistic & $17.4 \pm 14.1$ & $19.0 \pm 14.0$ & $17.1 \pm 13.0$ & $22.0 \pm 15.4$ & 0.13 \\
\hline NYHA III/IV & $119(51.5)$ & $21(50)^{b}$ & $65(43.6)^{b}$ & $33(82.5)^{a, c}$ & $<0.001$ \\
\hline Hypertension & $174(75.3)$ & $38(90.5)^{b}$ & $111(74.5)$ & $25(62.5)^{c}$ & 0.012 \\
\hline Diabetes & $87(37.7)$ & $17(40.5)$ & $56(37.6)$ & $14(35)$ & 0.88 \\
\hline PHT & $22(9.5)$ & $5(11.9)$ & $11(7.4)$ & $6(15)$ & 0.29 \\
\hline AF & $77(33.3)$ & $12(28.6)$ & $48(32.2)$ & $17(42.5)$ & 0.36 \\
\hline Prior $\mathrm{PCl}$ (< 12 months) & $60(26.1)$ & $7(16.7)$ & $42(28.4)$ & $11(27.5)$ & 0.31 \\
\hline CABG & $30(13)$ & $9(21.4)$ & $13(8.7)$ & $8(20)$ & 0.03 \\
\hline History of MI & $57(24.7)$ & $10(23.8)$ & $34(22.8)$ & $13(32.5)$ & 0.45 \\
\hline History of stroke with residual deficits & $33.7(3)$ & $10.2(4.8)$ & $18.3(2)$ & $5.2(5)$ & 0.48 \\
\hline $\mathrm{GFR}<30 \mathrm{ml} / \mathrm{min} / 1.73 \mathrm{~m}^{2}$ & $26(11.3)$ & $4(9.5)$ & $17(11.4)$ & $5(12.5)$ & 0.91 \\
\hline \multicolumn{6}{|l|}{ Echocardiography parameters: } \\
\hline $\operatorname{LVEF}(\%)$ & $51.7 \pm 14.4$ & $59.7 \pm 6.5^{a, b}$ & $54.5 \pm 12.8^{\mathrm{b}, \mathrm{c}}$ & $32.7 \pm 9.6^{a, c}$ & $<0.001$ \\
\hline LVEF $<40 \%$ & $57(24.7)$ & $O(0)^{a, b}$ & $25(16.8)^{b, c}$ & $32(80)^{a, c}$ & $<0.001$ \\
\hline AVAi $\left[\mathrm{cm}^{2} / \mathrm{m}^{2}\right]$ & $0.40 \pm 0.1$ & $0.47 \pm 0.1^{\mathrm{a}}$ & $0.37 \pm 0.1^{c}$ & $0.42 \pm 0.2$ & $<0.001$ \\
\hline $\mathrm{PGmax}[\mathrm{mm} \mathrm{Hg}]$ & $77.9 \pm 28.2$ & $56.4 \pm 15.5^{\mathrm{a}}$ & $91.6 \pm 24.0^{b, c}$ & $49.6 \pm 15.5^{a}$ & $<0.001$ \\
\hline PGmean [mm Hg] & $47.3 \pm 18.4$ & $31.9 \pm 7.2^{\mathrm{a}}$ & $56.8 \pm 15.1^{\mathrm{b}, \mathrm{c}}$ & $28.0 \pm 9.5^{\mathrm{a}}$ & $<0.001$ \\
\hline \multicolumn{6}{|l|}{ Procedural parameters: } \\
\hline Non-femoral access & $42(18.2)$ & $6(14.3)$ & $30(20.1)$ & $6(15)$ & 0.58 \\
\hline Self-expandable valve & $155(67.1)$ & $30(71.4)$ & $95(63.8)$ & $30(75)$ & 0.33 \\
\hline Major and life-threatening bleeding & $23(10)$ & $6(14.3)$ & $13(8.7)$ & $4(10)$ & 0.57 \\
\hline Stroke/TIA & $11(4.8)$ & $2(4.8)$ & $6(4)$ & $3(7.5)$ & 0.66 \\
\hline
\end{tabular}

${ }^{a} p<0.05$ vs. HGAS, ${ }^{b} p<0.05$ vs. CLF-LGAS, ${ }^{c} p<0.05$ vs. $p$ LF-LGAS. Continuous variables are shown as a mean \pm standard deviation, categorical variables are presented as absolute number (percentages). AF-atrial fibrillation, AVAi - aortic valve area index, BMI - body mass index, BSA - body surface area, CABG - coronary artery bypass graft, REF-LGAS - reduced ejection fraction, low-gradient aortic stenosis, GFR - glomerular filtration rate, HGAS - high-gradient aortic stenosis, CLF-LGAS classical low-flow, low-gradient aortic stenosis, LGAS - low-gradient aortic stenosis, LVEF-left ventricular ejection fraction, MI - myocardial infarction, MPG - mean pressure gradient, NYHA - New York Heart Association, pLF-LGAS - paradoxical low-flow, low-gradient aortic stenosis, PCI - percutaneous coronary intervention, $P G$ - pressure gradient, PHT - pulmonary hypertension, TIA - transient ischaemic attack.

were less symptomatic than the CLF-LGAS group and had higher LVEF than the HGAS patients.

In terms of age, the studies reporting on LF-LGAS are conflicting, some - especially those originating from surgical experiences - showing patients with paradoxical LF-LGAS to be older [10-12], whereas those investigating the issue from the transcatheter point of view do not detect such association $[9,13]$ or document pLF-LGAS subjects to be younger than HGAS subjects [14]. This is most likely due to the selection bias resulting from the relative novelty of the PLF-LGAS concept and possibility of delayed diagnosis of these patients in the early phases of surgical experience as well as the fact that the European guidelines only recognized pLF-LGAS as being an important part of the AS universe in 2012.

In terms of higher prevalence of hypertension within the pLF-LGAS group and more pronounced symptoms among the CLF-LGAS patients, our finding are in line with the majority of previous reports. Presence of hypertension is postulated as one of the major factors resulting in restrictive filling of the left ventricle, which along with the high arterial impedance results in a low-flow status. Similarly, the intensity of the symptoms in patients with 
CLF-LGAS is a result of the intertwined effect of prior heart dysfunction and aortic stenosis.

Higher ejection fraction among patients with pLFLGAS as compared to the HGAS group may be explained by the study design. Following the majority of investigators, we did not isolate from our population a group of patients with high gradient and reduced EF. This particular cohort, in which the decrease in EF is most likely reversible and results from preload and afterload mismatch due to substantial valvular obstruction, has not been proven to have significantly greater mortality than HGAS [9] and does not seem to be an autonomous entity, but rather an advanced form of HGAS. Nonetheless, incorporating patients with a high transvalvular gradient and decreased EF into the HGAS group may have influenced the mean EF in this cohort.

\section{Impact on mortality}

In our study, presence of paradoxical LF-LGAS did not influence the short- or mid-term survival as compared to HGAS and CLF-LGAS. Similarly, it was not found to be an independent predictor of mortality in the multivariate analysis. This can be attributed to the relatively short follow-up period as well as the small study sample. On the other hand, papers reporting on paradoxical LF-LGAS are not unanimous as to whether the diagnosis of pLF-
LGAS worsens the survival in this cohort of patients. In the study by Abramowitz et al., patients with LF-LGAS had increased mortality following TAVI compared with

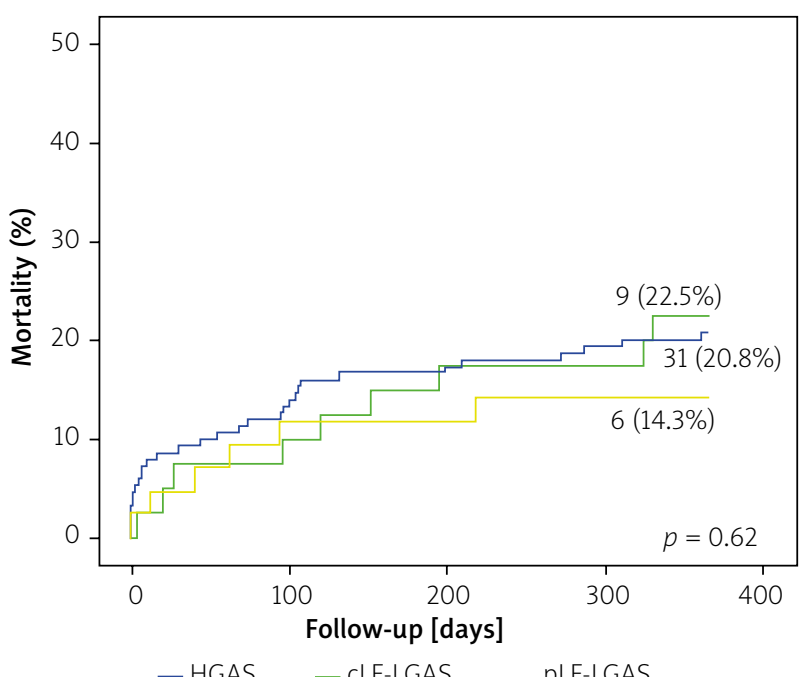

Figure 2. Kaplan-Meier curves comparing 1-year mortality rate between different subsets of aortic stenosis patients treated

CLF-LGAS - classical low-flow, low-gradient aortic stenosis, HGAS - high-gradient aortic stenosis, pLF-LGAS - paradoxical low-flow, low-gradient aortic stenosis.

Table II. Uni- and multivariate analysis of possible predictors of mortality among AS patients

\begin{tabular}{|c|c|c|c|c|c|c|}
\hline Variables & Odds ratio (OR) & $\begin{array}{l}\text { Confidence } \\
\text { interval (CI) }\end{array}$ & $P$-value & Odds ratio (OR) & $\begin{array}{c}\text { Confidence } \\
\text { intervals (Cl) }\end{array}$ & $P$-value \\
\hline Age [years] & 0.98 & $0.94-1.01$ & 0.18 & & & \\
\hline Female sex & 0.74 & 0.74 & 0.32 & & & \\
\hline $\mathrm{BMI}\left[\mathrm{kg} / \mathrm{m}^{2}\right]$ & 0.97 & $0.91-1.04$ & 0.4 & & & \\
\hline NYHA III/IV & 1.43 & $0.8-2.58$ & 0.23 & & & \\
\hline Diabetes & 0.68 & $0.36-1.28$ & 0.23 & & & \\
\hline Hypertension & 0.64 & $0.35-1.19$ & 0.16 & & & \\
\hline AF & 0.79 & $0.42-1.5$ & 0.79 & & & \\
\hline History of MI & 0.53 & $0.24-1.19$ & 0.13 & & & \\
\hline History of stroke with residual deficits & 0.66 & $0.08-5.65$ & 0.71 & & & \\
\hline LVEF (\%) & 0.99 & 0.98-1.1.02 & 0.76 & & & \\
\hline LVEF $<40 \%$ & 0.83 & $0.41-1.67$ & 0.6 & & & \\
\hline PGmean [mm Hg] & 1 & $0.99-1.02$ & 0.85 & & & \\
\hline PGmax [mm Hg] & 1 & $0.99-1.01$ & 0.75 & & & \\
\hline $\mathrm{GFR}<30 \mathrm{ml} / \mathrm{min} / 1.73 \mathrm{~m}^{2}$ & 2.31 & $1.1-4.79$ & 0.03 & 2.1 & $1.01-4.37$ & 0.046 \\
\hline HGAS & 1.18 & $0.64-2.18$ & 0.61 & & & \\
\hline cLF-LGAS & 1.07 & $0.74-1.53$ & 0.73 & & & \\
\hline pLF-LGAS & 0.87 & $0.65-1.16$ & 0.34 & & & \\
\hline
\end{tabular}

AF-atrial fibrillation, BMI-body mass index, CLF-LGAS-reduced ejection fraction-low-gradient aortic stenosis, GFR-glomerular filtration rate, HGAS - high-gradient aortic stenosis, LF-LGAS - low-flow-low-gradient aortic stenosis, LGAS - low-gradient aortic stenosis, LVEF-left ventricular ejection fraction, MI - myocardial infarction, PGmean - mean pressure gradient, PGmax - maximal pressure gradient, NYHA - New York Heart Association, pLF-LGAS - preserved ejection fraction - low-gradient aortic stenosis. 
those with HGAS and those with NF-LGAS. Among patients with LF-LGAS, CLF-LGAS patients had increased cardiovascular mortality compared to those with pLF-LGAS (27.3\% vs. $19 \%$, respectively) [15]. Other recent studies also document an association of low flow with higher incidence of cardiovascular mortality [16]. In the subanalysis of the PARTNER trial [17] patients with low-flow AS (a pooled cohort of patients with CLF-LGAS and pLFLGAS) had significantly higher 2-year mortality than subjects with normal flow AS (47.1\% vs. $33.7 \%, p<0.0001)$. However, there was no difference in mortality between CLF-LGAS and pLF-LGAS (48.7\% vs. $46.1 \%, p=0.7$ ). In the study by Puls et al., patients with pLF-LGAS had significantly higher 1-year mortality (31.2\% vs. $14.3 \%$, $p<0.05$ ) and shorter median survival (3.3 vs. 5.1 years, $p<0.05)$ than the HGAS with preserved EF group, but not compared to the HGAS with decreased EF cohort (median survival 3.3 vs. 4.6 years). On the other hand, pLF-LGAS was not a predictor of all-cause and cardiovascular mortality in the multivariate analysis, nor did it predict cardiac-related hospitalizations and MACCE [18]. Finally, in a subgroup analysis from the GARY registry [14] based on a real-world cohort of 2863 TAVI patients, mortality in pLF-LGAS was comparable to HGAS (in-hospital: $5.3 \%$ vs. $4.9 \%, p=0.68$, 1 -year: $22.3 \%$ vs. $19.8 \%$, $p=0.19)$. In our study, the mortality rate among patients with pLF-LGAS, HGAS and CLF-LGAS was 6 (14.3\%), $31(20.8 \%)$ and $9(22.5 \%)$, respectively. The numerically lower mortality among pLF-LGAS compared to CLF-LGAS patients may be explained by potential advantages of the new-generation devices in the treatment of this cohort of TAVI patients. However, this difference may also be a result of a small sample size.

The only independent predictor of mortality in our study was CKD, with GFR below $30 \mathrm{ml} / \mathrm{min} / 1.73 \mathrm{~m}^{2}$ resulting in an over two-fold increase in mortality in this subgroup of patients - a finding which is in line with postulated risk factors among TAVI patients. Tamburino et al. reported chronic kidney disease to be an independent predictor of mortality between 30 days and 1 year with a hazard ratio of 2.53 [19]. In an analysis by Levi et al. encompassing 1204 patients from 10 centres in Europe, Japan, and Israel, advanced CKD was associated with a 2-fold increase in the adjusted risk of 1-year allcause death $(p<0.001)$, and a 1.9-fold increase in cardiovascular death $(p=0.016)$ [20].

\section{A word on classical low-flow, low-gradient aortic stenosis}

Meticulous assessment of the severity and type of AS as well as accompanying patients' individual characteristics is crucial for optimal qualification, therapeutic management and future outcomes in the setting of transcatheter aortic valve implantation. It has been postulated in previous reports that patients with LF-LGAS, especially those with CLF-LGAS, have poor quality of life $[7,10,21]$. Although the most typical form of AS is HGAS, patients with severe CLF-LGAS (5-10\% of cases) and pLF-LGAS (10-25\% of cases) combined represent an important proportion of patients with severe AS [22]. In the literature, there is a consensus that CLF-LGAS patients exhibit more comorbidities, including $C A D$, peripheral vascular disease (PHD), CKD, previous myocardial infarction (MI) and prior percutaneous coronary intervention $(\mathrm{PCl})$ or coronary artery bypass graft (CABG), compared to other AS subtypes $[10,23]$. Such patients are classically characterized by a dilated LV with markedly decreased LV systolic function, most often due to ischaemic heart disease and/or to afterload mismatch [22, 24]. In particular, a high proportion ( $46 \%$ to $79 \%$ ) of those patients have concomitant CAD [18, 21].

In our study, despite the lack of noticeable difference in the percentage of patients with a history of MI, higher presence of advanced CAD in the CLF-LGAS group was demonstrated by a markedly larger pool of post-CABG patients as compared to HGAS. Furthermore, a significantly higher proportion of patients with CLF-LGAS were heavily symptomatic at baseline due to the combined impact of underlying heart dysfunction and severe AS. Almost $80 \%$ of our patients with CLF-LGAS had moderate and severe, whereas only $20 \%$ had mild LV systolic dysfunction. Despite being numerically higher, the mortality in this group was not significantly greater as compared to the paradoxical LF-LGAS and HGAS groups. This is most likely a result of a relatively small group and limited follow-up period as well as a substantial number of early events in the HGAS group, which more often correspond to acute and sub-acute complications of TAVI (e.g. bleeding, vascular injury, infections) and may be less related to the heart dysfunction.

\section{Study limitations}

The main limitation of the study is its retrospective design and limited study sample. Therefore no definite conclusions can be drawn. Moreover, some variables previously reported as being risk factors for the disease or disease progression (i.e., LV longitudinal strain, left atrial volume, B-type natriuretic peptide and valve calcification) were not measured in this study, thus limiting the characterization of study groups.

\section{Conclusions}

Paradoxical low-flow low-gradient aortic stenosis is common among subjects undergoing transcatheter aortic valve implantation. Patients with paradoxical AS are younger, more often burdened with hypertension and have higher EF than the HGAS population, while being less symptomatic than the CLF-LGAS group. Presence of pLF-LGAS does not seem to affect short- and mid-term survival. Severe renal failure is an independent predictor of mortality after TAVI. 


\section{Conflict of interest}

The authors declare no conflict of interest.

\section{References}

1. Rosenhek R, Zilberszac R, Schemper M, et al. Natural history of very severe aortic stenosis. Circulation 2010; 121: 151-6.

2. Kurtz CE, Otto CM. Aortic stenosis: clinical aspects of diagnosis and management, with 10 illustrative case reports from a 25year experience. Medicine (Baltimore) 2010; 89: 349-79.

3. Vahanian A, Alfieri O, Andreotti F, et al. Guidelines on the management of valvular heart disease (version 2012). Eur Heart J 2012; 33: 2451-96.

4. Baumgartner H, Falk V, Bax JJ, et al. 2017 ESC/EACTS Guidelines for the management of valvular heart disease. Eur Heart J 2017; 36: 2739-91.

5. Nishimura, RA, Otto CM, Bonow RO, et al. 2014 AHA/ACC guideline for the management of patients with valvular heart disease: executive summary: a report of the American College of Cardiology/American Heart Association Task Force on Practice Guidelines. Circulation 2014; 63: 2438-88.

6. Mehran R, Rao SV, Bhatt DL, at al. Standardized bleeding definitions for cardiovascular clinical trials: a consensus report from the Bleeding Academic Research Consortium. Circulation 2011; 123: 2736-47.

7. Clavel MA, Fuchs C, Burwash IG, et al. Predictors of outcomes in low-flow, low-gradient aortic stenosis: results of the multicenter TOPAS Study. Circulation 2008; 118: 234-42.

8. Barasch E, Fan D, Chukwu EO, et al. Severe isolated aortic stenosis with normal left ventricular systolic function and low transvalvular gradients: pathophysiologic and prognostic insights. J Heart Valve Dis 2008; 17: 81-8.

9. Lancellotti P, Donal E, Magne J, et al. Impact of global left ventricular afterload on left ventricular function in asymptomatic severe aortic stenosis: a two-dimensional speckle-tracking study. Eur J Echocardiogr 2010; 11: 537-43.

10. Clavel MA, Berthelot-Richer M, Le Ven F, et al. Impact of classic and paradoxical low flow on survival after aortic valve replacement for severe aortic stenosis. J Am Coll Cardiol 2015; 65: 645-53.

11. Lopez-Marco A, Miller H, Kumar P, et al. Outcome of isolated aortic valve replacement in patients with classic and paradoxical low-flow, low-gradient aortic stenosis. J Thorac Cardiovasc Surg 2017; 154: 435-42.

12. Herrmann S, Stork S, Niemann M, et al. Low-gradient aortic valve stenosis: myocardial fibrosis and its influence on function and outcome. J Am Coll Cardiol 2011; 58: 402-12.

13. Kataoka A, Watanabe $Y$, Kozuma K, et al. Prognostic impact of low-flow severe aortic stenosis in small-body patients undergoing TAVR: The OCEAN-TAVI Registry. JACC Cardiovasc Imaging 2018; 11: 659-69.

14. Lauten A, Figulla HR, Möllmann H, et al. TAVI for low-flow, lowgradient severe aortic stenosis with preserved or reduced ejection fraction: a subgroup analysis from the German Aortic Valve Registry (GARY). Eurolntervention 2014; 10: 850-9.

15. Abramowitz Y, Chakravarty T, Pibarot P, et al. Characteristics and outcome following transcatheter aortic valve replacement in patients with severe aortic stenosis with low flow. Eurolntervention 2017; 13: e1428-35.

16. Mangner N, Stachel G, Woitek F, et al. Predictors of mortality and symptomatic outcome of patients with low-flow severe aortic stenosis undergoing transcatheter aortic valve replace- ment. J Am Heart Assoc 2018; 7: pii: e007977. doi: 10.1161/ JAHA.117.007977.

17. Herrmann HC, Pibarot P, Hueter I, et al. Predictors of mortality and outcomes of therapy in low-flow severe aortic stenosis: a Placement of Aortic Transcatheter Valves (PARTNER) trial analysis. Circulation 2013; 127: 2316-26.

18. Puls M, Korte KP, Bleckmann A, et al. Long-term outcomes after TAVI in patients with different types of aortic stenosis: the conundrum of low flow, low gradient and low ejection fraction. Eurolntervention 2017; 13: 286-93.

19. Tamburino C, Capodanno D, Ramondo A, et al. Incidence and predictors of early and late mortality after transcatheter aortic valve implantation in 663 patients with severe aortic stenosis. Circulation 2011; 123: 299-308.

20. Levi A, Codner P, Masalha A, et al. Predictors of 1-year mortality after transcatheter aortic valve implantation in patients with and without advanced chronic kidney disease. Am J Cardiol 2017; 120: 2025-30.

21. Tribouilloy C, Rusinaru D, Marechaux S, et al. Low-gradient, lowflow severe aortic stenosis with preserved left ventricular ejection fraction. J Am Coll Cardiol 2015; 65: 55-66.

22. Pibarot P, Dumesnil JG. Low-flow low-gradient aortic stenosis with normal and depressed left ventricular ejection fraction. J Am Coll Cardiol 2012; 60: 1845-53.

23. Le Ven F, Freeman $M$, Webb J, et al. Impact of low flow on the outcome of high-risk patients undergoing transcatheter aortic valve replacement. J Am Coll Cardiol 2013; 62: 782-8.

24. Monin JL, Quere JP, Monchi M, et al. Low-gradient aortic stenosis: operative risk stratification and predictors for long-term outcome: a multicenter study using dobutamine stress hemodynamics. Circulation 2003; 108: 319-24. 